\title{
KELEA Activation of Water and Other Fluids for Health, Agriculture and Industry
}

\author{
W. John Martin \\ Institute of Progressive Medicine, South Pasadena, USA \\ Email: wjohnmartin@ccid.org
}

Received 6 November 2015; accepted 22 November 2015; published 25 November 2015

Copyright (C) 2015 by author and Scientific Research Publishing Inc.

This work is licensed under the Creative Commons Attribution-NonCommercial International License (CC BY-NC).

http://creativecommons.org/licenses/by-nc/4.0/

(c) (i) (8) Open Access

\begin{abstract}
Water is a critical component in the functioning of the earth and of all living creatures. An important insight into the variable physical and biological properties of water has been provided by studies consistent with an external force termed KELEA (kinetic energy limiting electrostatic attraction) that weakens the intermolecular bonding of water molecules. Simple methods are described in this paper for producing and testing KELEA activated water. A summary is also provided of many major applications of KELEA activated water in health, agriculture and industry. While several of the proposed applications require more rigorous scientific documentation and further optimization, collectively they present the compelling opportunity for KELEA activated water to immediately transform medical, agricultural and industrial practices. A coordinated endeavor is proposed to better understand the science of KELEA and to develop simplified protocols for utilizing KELEA activated water and other fluids in the presently described and likely numerous additional applications. This ambitious undertaking envisions the cooperation among a range of specialized philanthropic organizations, working within different countries and with the strong support of national governments.
\end{abstract}

\section{Keywords}

KELEA, Water, Philanthropy, Insufficiency of Cellular Energy, Alternative Cellular Energy, ACE Water $^{\mathrm{TM}}$, Dipolar Compounds, Enerceutical ${ }^{\mathrm{TM}}$, Waterceutical ${ }^{\mathrm{TM}}$, Homeopathy, Gasoline

\section{Introduction}

Research over the last several years has identified variable physical qualities of water, which are attributable to weakening of the intermolecular hydrogen bonding among water molecules [1] [2]. It is proposed that the wea- 
kening occurs from the absorption of an environmental force called KELEA (kinetic energy limiting electrostatic attraction). This proposed fundamental force of nature is thought to basically prevent the fusion and potential annihilation of electrostatically attracted opposite electrical charges. KELEA is apparently attracted to separated electrical charges, whether existing on dipolar molecules or created by electrical devices. Through oscillations of the dipolar molecules or the on-off switching of the electrical devices, the attracted and concentrated KELEA can be released in a manner that can affect nearby water. Loosening occurs in the electrostatic attraction that normally mediates the relatively stable positioning of hydrogen-bonded water molecules. This allows for increased movements (kinetic activity) of the water molecules. Moreover, if sufficient electrical charge separation is achieved in the water, there can be a continuing attraction of KELEA, with internal energy transfers occurring within the essentially de-structured water [3]. This can lead to progressively greater activation of the water that can extend to added water and even to nearby water. Secondary water activation explains the capacity of water remaining highly active even during sequential dilutions, as employed in homeopathy [4].

\section{Methods of Producing KELEA Activated Water}

The two basic approaches to KELEA activation of water and other fluids are: i) Adding certain dipolar and other compounds with separated electrical charges to the fluid; and ii) exposing the fluid to an energy field that can lead to increased local levels of KELEA [5]. The author has personally tested many of the water activating methods described below. For convenience of presentation, the water activating materials are broadly grouped into 5 categories (Table 1). The first category applies to common, earth-derived, mineral containing dipolar compounds including humic/fulvic acids, zeolites, activated charcoal, mica, gypsum, various other ceramics, volcanic rocks, shungite (a product from Russia), and granular magnesium oxide. This category also includes certain geographic locations, such as deep volcanic springs, from which bottles of naturally activated drinking water are now available. The second category of water activating chemicals relates to various pharmaceuticals, with apparent clinical benefits beyond their biochemical activities. Examples include procaine and xylocaine (Lidocaine), ascorbic acid (vitamin C), niacin (vitamin B3), Dilantin, colloidal silver and possibly monoclonal immunoglobulins. The third category applies to food products, such as Moringa oleifera, Ashitaba angelica and cocoa (baked cacao). This category also extends to tinctures and extracts derived from natural organic products. Three examples of the latter are the terpene-rich sap of certain Japanese trees, contained in the product HB-101; Folium pX from the bark, needles and cones of pine trees and d-limonene from orange peel. Several herbal tinctures included in this category are commonly used in homeopathic formulations. A fourth category of water activating components applies to different gases. Their ability to attract KELEA is presumably a result of the charge separation between the nucleus and orbiting electrons on the gas molecules. Water activating gases include hydrogen, ozone, chlorine dioxide, some noble gases and Brown's gas [6] (a water electrolysis induced mixture of hydrogen, oxygen and activated water vapor). Hydrogen and chlorine dioxide gases can be generated from components added to water, while electrolysis can be performed using electrolytes with additional, potent, water activating properties. An example is electrolyzed water comprising a copper and silver citrate solution, shown by the author and a colleague to be effective in wound healing. The fifth category is based on the simple premise that once water or other fluids are sufficiently activated, they can transfer the activation to added fluids in a progressive manner. The term enerceutical ${ }^{\mathrm{TM}}$ has been suggested for compounds that are able to transfer KELEA into water or, alternatively, if consumed can directly enhance the kinetic activity of the body's fluids.

An important feature of enerceuticals ${ }^{\mathrm{TM}}$ is that if the water becomes sufficiently activated, the compounds do

\begin{tabular}{ccc}
\hline \multicolumn{3}{l}{ Table 1. Categorization and examples of materials useful for activating water. } \\
\hline Category & Description & Examples \\
\hline 1 & Earth-derived & Humic/fulvic acids, zeolites, magnesium oxide \\
2 & Pharmaceuticals & Lidocaine, Dilantin, ascorbic acid, niacin \\
3 & Plant-derived & Moringa oleifera, cocoa, herbal tinctures \\
4 & Gases & Hydrogen, ozone, chlorine dioxide, Brown's gas \\
5 & Activated fluids & Previously activated water and/or ethanol \\
\hline
\end{tabular}


not necessarily need to be retained in the water. Specifically, if the activating compounds are insoluble, as with pellets of heated ceramics, ground volcanic rock, magnesium oxide granules, etc., a container holding the materials can simply be retrieved from the activated water. The materials can be reused on numerous occasions in the activation of additional water. If the insoluble water activating material is not in containers, it can still be separated from the water by simply decanting the activated water and replacing with regular water. Soluble water activating compounds can be removed from the water by zero residue filtration or by repeated dilution. One to two days of exposure to the activating compounds (commonly ranging from approximately $0.001 \%$ to $0.01 \%$ for soluble materials and up to $10.0 \%$ for insoluble granules and pellets) is adequate for effective water activation. Continually supplied gases can also take time to activate water, especially since bubbled gases are quickly released from the water to the atmosphere.

The second basic approach to activating water is via the formation of concentrated KELEA energy fields within the vicinity of the water. These energy fields can be developed by the rapid on-off switching of highly chargeable electrical devices. Historical examples of such devices include the Violet Ray of Edgar Cayce [7], the Beam Ray of Royal Raymond Rife [8] and the multi-wave oscillator of Lakhovsky [9]. Many of these devices can also create ozone and negative ions, which may also contribute to water activation. As noted above, electrolysis can activate water as well as creating vaporized water molecules (the unique component of Brown's gas). Converging and opposing light beams can also attract and concentrate KELEA from the environment [10], as can coils of electric cord transmitting direct current (DC) [11]. Such coils can also be submerged into the water to be activated. Additional methods of water activation include enclosures with alternating conducting and insulating layers (orgone chamber of Reich [12]), heated and differentially cut series of seven aluminum tubes (Intrasound of Roehrich [13]), and an antenna-based device termed Aquapol, used to reverse the upward flow of ground water into the foundations of buildings (developed by Mohorn [14]). These last three examples are notable in not requiring connection to a defined energy supply. They may, however, acquire separated charges based upon the earth's negative charge in comparison to that of the upper atmosphere. The lack of dependence on a conventional energy supply also exists with pyramids and other geometric structures that can apparently increase the dynamic quality of water, possibly by affecting the natural flow of KELEA. Sunlight passing through cobalt blue glass, as first described by Augustus Pleasanton in 1877, may have a similar effect [15]. Rotating magnets can establish fluctuating electrostatic fields, as can piezoelectric oscillators.

The most biologically relevant of all of the KELEA attracting mechanism may well be the electrical charge separation that occurs in the membranes of all living cells. Indeed, this antenna-like capacity may be amplified by some of the brain's coordinated electrical activity, as reflected in the electroencephalogram (EEG) [16]. A similar antenna role may extend to the electrical activities of muscles, including the heart. An extrapolation from the concept that humans may naturally be able to attract KELEA is that certain individuals and possibly animals may also be able to transmit KELEA to others, with a beneficial outcome. Conversely, certain individuals appear to exert a negative influence on the emotional wellbeing of others, possibly by withdrawing KELEA.

As with most natural phenomena, KELEA activation is likely to be counteracted or balanced by other influences. It is noteworthy, for example, that homeopaths ask that their remedies not be exposed to X-rays or to microwaves, believing that these energies diminish the therapeutic value of the remedies. They also believe it is easier to activate distilled rather than regular water, an observation confirmed by the author. The possible influence of external weather patterns on the ease of activating water has also been contemplated [1]. The greater volatility of activated molecules can explain the reduction in biological activity over time in water stored in unsealed containers. This problem arose in the loss of the earlier effectiveness of using ultraviolet light (UV) illumination of activated water applied to neutral red treated paper towel laid onto the skin of children with autism [17]. The activated water was stored in a closed but not sealed container and was repeatedly subjected to vigorous jolting (succession) before obtaining aliquots for testing.

Pollack describes water that is adjacent to hydrophobic membranes as being activated but many of its properties are the reverse of those that most people associate with activated water [18]. DeMeo induced similar water by placing the water under a cellulose acetate cover [19]. When tested on plants, this water had a marked inhibitory effect, which was lost when the water was removed from being near the cellulose acetate. It would not be surprising, if very tall trees used a mechanism that could strengthen the cohesive hydrogen bonding of water molecules, as a means of assuring that ground water reaches the uppermost regions of the trees. The terms activated water, ACE Water ${ }^{\mathrm{TM}}$ and Waterceuticals ${ }^{\mathrm{TM}}$, should be reserved for water shown to have greatly increased 
kinetic activity with enhanced biological activities that are not related to temperature and pressure.

\section{Properties and Testing of KELEA Activated Water}

The physical changes in KELEA activated water extend beyond its greater kinetic activity. The important changes include lowered surface tension, lowered specific heat, lowered freezing and boiling points, increased volatility and more efficient support of various biological activities [20]. Activated water attaches less to its own molecules and even less so to molecules of other substances. Hence, there is less solubility of various substances in activated water. Indeed, some contaminants will noticeably precipitate out from water during the activation process. Conversely, activated water can assist in the dispersal of hydrogen bonded aggregates, such as scale formation in metal pipes, into smaller particles that are easily washed away. Moreover, the lower surface tension allows activated water to better penetrate into spaces that are less permeable to regular water, adding to its more efficient removal of debris. The reduced intermolecular bonding and greater mobility of the resulting smaller clusters of water molecules is seen in the more rapid and less variable timing of realignment of magnetically oriented water subject to a pulsed radio wave, as used in nuclear magnetic resonance (NMR). There is also an increase in both the absorption and emission of far-infrared radiation by the dynamically changing hydrogen bonds in the activated water. These last two parameters are measurable using complex methodology. A robust testing method, based on the increased volatility of activated water, is to measure the rate of weight reduction in tightly closed but not completely sealed small containers [10] [11]. This approach can also be used to test for the water activating capacity of various substances, including activated water when added to test samples of distilled water. A similar approach can be used to test for water activating energy fields. Other simple positive screening tests for water activation include i) the ease of initiating and the duration of vortex movement in spun containers of the water, ii) very fine condensation droplets above the water line in the containers, iii) lowered convexity of individual water droplets, and iv) the pattern seen when particles of neutral red dye are sprinkled onto the water. This can range from essentially stable particles laying on the surface of the water to particles displaying to-andfro linear movements. The particles will also sink below the surface of activated water due to its lowered surface tension.

Ethanol is less miscible in activated water and mixtures of these activated fluids will commonly separate into two distinct layers. The reduced freezing point slows the formation of ice crystals accounting for the formation of more symmetrical ice crystals, as widely reported by the late Dr. Masaru Emoto using his water [21].

The amount of heat required to raise the temperature of one gram of distilled water by one degree centigrade $\left(1{ }^{\circ} \mathrm{C}\right)$ is defined as one calorie. This value referred to as "specific heat" was assumed to be constant for water. Research, however, has shown that KELEA activated water has a lower specific heat value [2]. In other words, a calorie will raise the temperature of a gram of activated water by more than a $1^{\circ} \mathrm{C}$. Direct testing of this feature needs to consider the increased volatility of activated water, with some heat loss due to evaporation. In practice, it is easier to discern the lower boiling and freezing points of activated water. Essentially, less heat input is required to generate steam from activated water, and incomplete freezing can occur at $0^{\circ} \mathrm{C}$. A difficulty in establishing units of activation is that other factors can have confounding effects on various measurements. These influences include temperature, pressure and other substances present in the water.

The ultimate test of water activation is the degree of improvement achievable in the particular function for which the water is being used. The most remarkable of these is the capacity of activated water to enhance a wide range of biological functions. Enzymes are more active within activated water and cellular movements are increased. Activated water is essentially an added energy source for living cells. On the basis that Nature has photosynthesis as its first energy pathway and nutrient metabolism as the second, the cellular energy acquired through KELEA has been designated as the third or alternative cellular energy (ACE) pathway [5]. Accordingly, in the biological context, activated water has become synonymous with ACE Water. The following sections will briefly outline some of the immediately available major applications of activated water in health, agriculture and industry.

\section{Health Benefits of KELEA Activated Water}

The major medical uses of KELEA activated water will be to treat illnesses due to an insufficiency of cellular energy (ICE). In principle, the use of activated water can be extended to the prevention of illnesses, which might 
otherwise develop as a result of ICE. Food metabolism is primarily the process of oxidative phosphorylation [22]. Complex food molecules are digested into basic chemical units that can enter the Krebs cycle. This cycle enables the mitochondria to transfer separated electrons across a lipid membrane, thereby creating an electrostatic gradient. The electrons return back across the membrane in a manner that results in the synthesis of adenosine triphosphate (ATP) from lower energy precursor molecules. The chemical energy within ATP is utilized in the majority of biochemical reactions within living cells. These biochemical reactions occur within water and their efficiency is likely directly influenced by the kinetic quality of the water.

ICE resulting from a limitation of the second or food-derived cellular energy pathway is the basis for the vast majority of common, chronic illnesses affecting mankind. The generation of cellular energy from food can be adversely affected by a variety of disease processes ranging from an inadequate delivery of oxygen and nutrients to the tissues; inefficient cellular metabolic processes; and increased energy demands caused by infections or required for tissue repair and for normal growth and development (Table 2).

The somewhat exhaustive listings of medical applications included in Table 3, are all consistent with being fundamentally caused by ICE and, therefore, suitable for clinical testing using KELEA activated water. Empirical data exist for the benefit of consuming activated water and/or enerceuticals ${ }^{\mathrm{TM}}$ in many of these common illnesses. Some authors assert that virtually all patients are chronically dehydrated and that clinical improvements can be anticipated from increasing daily water consumption [23]. Clinical studies assessing the value of water consumption are typically not rigorously pursued or promoted when compared with studies using patented pharmaceuticals. Nor has there been a distinction between using activated versus regular water. Detailed clinical studies will help to make this distinction by including "blinded" comparisons of consuming KELEA activated water with equivalent quantities of regular water. Government sponsored studies on the health benefits of consuming activated water will provide a far more appropriate use of public monies than subsidizing the research and development of patented pharmaceutical drugs.

Of the many conditions listed in Table 3, chronic obstructive pulmonary disease (COPD) has the advantage of easily measured changes in resting $\mathrm{pO}_{2}$ level. Improvements in cardiovascular diseases can also be easily assessed by increased endurance of measured physical activities, such as climbing stairs or distances travelled without needing to rest or experiencing angina. It would seem that Type 2 diabetes is a relatively easy illness to

\title{
Table 2. Reasons for Insufficiency of Cellular Energy (ICE) from food metabolism.
}

\author{
Nutritional deficiency \\ Inadequate oxygen uptake by the lungs \\ Reduced oxygen carrying capacity of the blood \\ Impaired tissue delivery of oxygen or nutrients \\ Inefficient metabolic pathways in the cells \\ Increased cellular energy demands
}

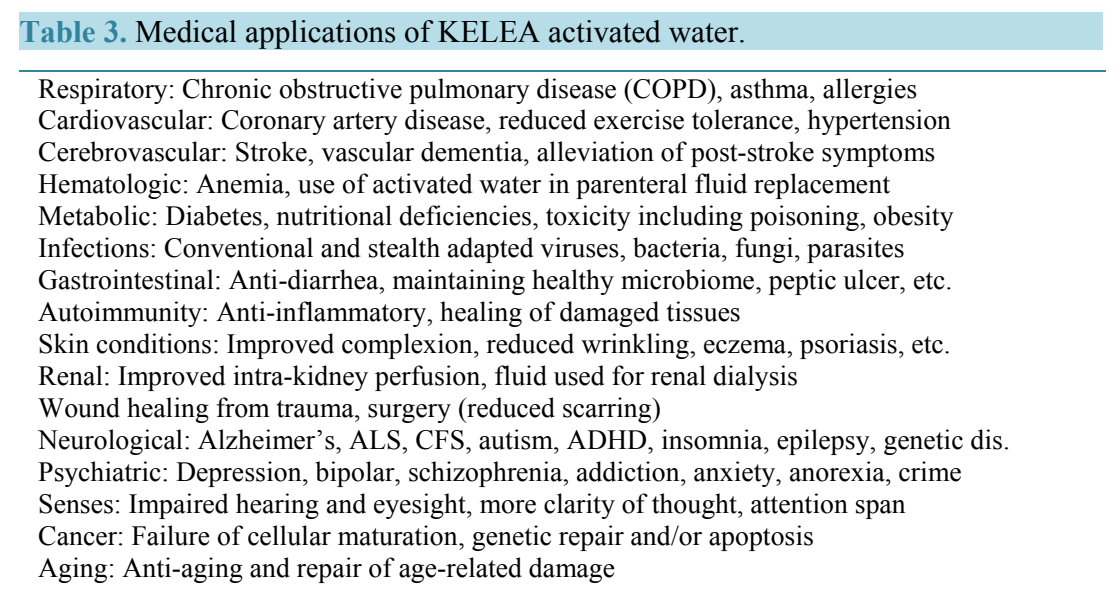


control with lifestyle changes. Although many of the suggested changes relate to reduced food intake, the author is aware of some rather remarkable results from simply drinking activated water. The effects have been so pronounced that the prior consumption of a glassful of activated water has offset the anticipated high elevation in glucose from a major meal. Long term improvement of Type 2 diabetes can be documented by a return to normal daily blood glucose levels with a lowering or abolishing of the need for added medications.

The uses of activated water in infectious diseases are particularly relevant to virus infections. The activated water can potentially act as an adjunct to and more importantly as an alternative to the immune system. It is commonly assumed that the body relies solely upon the immune system as its major defense against infections. Yet the effective immune recognition of viruses can be negated by a simple loss or mutation of the virus genes coding for the relatively few components normally targeted by the cellular immune system. This immune evasion mechanism has been termed stealth adaptation [24] [25]. Stealth adapted viruses are especially prone to induce symptomatic illness through their effects on the brain [26] [27]. Other viruses such as HIV directly destroy certain types of $\mathrm{T}$ lymphocytes, again rendering the immune system rather ineffective. Finally, some viruses can infect so rapidly that there is insufficient time for the immune system to mobilize or the viruses can sequester in locations beyond the range of the immune system. In these circumstances, the cell-virus relationship reverts to an ongoing competition for cellular energy resources. These resources can either go towards virus replication or towards the cells' ability to suppress the infecting virus.

Clinical studies have shown that the ACE pathway can assist the body in overcoming illnesses caused by stealth adapted viruses. Enhancing the ACE pathway has also shown benefits in treating HIV, herpes simplex virus (HSV), hepatitis viruses and rotavirus [28]-[30] and unpublished studies]. Support for this statement has come from studies on a water based product called Enercel ${ }^{\circledR}$. Although marketed elsewhere as HANSI (homeopathic activator of the system immune), the United States producer changed its name to Enercel, largely upon the author's observation of its suppression of the in vitro culture growth of stealth adapted viruses. In spite of being referred to as homeopathic, HANSI and some forms of Enercel have detectable levels of Lidocaine, a water acting dipolar enerceutical ${ }^{\mathrm{TM}}$. Rather than being promoted as unique proprietary therapies, Enercel and other clinically effective homeopathic formulations should be more openly presented as clinically validated examples of KELEA activated water [3].

Extracellular microbes, including pathogenic bacteria and fungi, may also be selectively targeted by KELEA activated water. One possible reason under study is that certain pathogenic microbes may have weaker defense mechanisms to avoid osmotic damage when compared to normal commensal microbes. The greater permeability of KELEA activated water, compared to regular water may, therefore, selectively damage pathogenic bacteria. It is also possible that weakened hydrogen bonding may also lead to the reduction of the activity of certain soluble toxins secreted by pathogenic microbes. It is noteworthy that Enercel is effective in the therapy of bacterial diarrhea [30] and tuberculosis [31].

Activating the ACE pathway can help limit the involvement of inflammation in response to infectious diseases. Beyond this, the ACE pathway may also be primarily anti-inflammatory. This is consistent with benefits seen with homeopathic formulations in the treatment of allergic disorders, such as asthma and of autoimmune diseases, such as systemic lupus erythematosus (SLE) and Hashimoto's thyroiditis. It would be interesting if an aspect of inflammation was to provide a source of nutrients retrieved from tissue cells killed in the inflammatory process.

Based on direct experience and extrapolating from published studies using homeopathic formulations, activated water can be beneficial even beyond the many illnesses directly attributed to ICE. For example, increased activated water consumption correlates with decreased food intake (unpublished). Possibly, a person may overeat in an effort seeking to remedy a deficiency in the ACE pathway. Increased consumption of activated water may, thereby, help address the rapidly growing obesity epidemic. This benefit would lead to reduced food costs. There can also be savings on the amount of water necessary to be consumed. Empirical observations strongly suggest that urination occurs sooner after consuming regular tap water than after consuming activated tap water. This is consistent with more of the consumed regular water remaining within the vasculature with less whole body distribution than achieved with the consumption of activated water. In addition to its many other advantages, activated water may, therefore, help in situations of water shortage, including allowing for less need for military troops to carry water into battle zones.

Enercel was likely also effective in treating children with tropical diarrhea because it could enhance the water absorbing capacity of gastrointestinal cells [30]. As mentioned earlier, activated water may also impact on the microbiome through selective toxicity of the more permeable water for osmotically challenged bacteria. Acti- 
vated water may also enhance repair mechanisms of peptic ulcers, as it has been shown to promote the repair of traumatic wounds, including surgery. For example, a KELEA activated water was being used primarily for its anti-bacteria effects in rinsing the exposed abdominal cavity of a goat following caesarian section. The solution was regularly applied to the sutured abdominal incision. Remarkably, no uterine adhesions developed post operatively and after only 3 weeks, the minimal skin scarring appeared as if it the incision was many months old [32].

Anecdotal stories of expedited wound healing are noteworthy because of the apparent absence of the usual inflammatory reaction that induces scarring. It appears as if inflammation, with its associated scarring, is a backup mechanism that occurs when natural restorative healing fails. As noted above, the inflammation of autoimmune diseases may similarly become suppressed in those consuming activated water or boosting their ACE pathway by other means.

The reported anti-inflammatory responses extend to noted improvements in psoriasis, eczema, cellulite, bee stings and other skin disorders. Moreover, activated water probably increases hydration of the skin leading to noticeably less wrinkling and may help in the elimination of pigmented debris in aged spots. Although as mentioned earlier, consuming activated water can lead to less urgency to urinate, its increased permeability may help counter renal insufficiency caused by inadequate blood perfusion of damaged kidneys.

Considerable anecdotal evidence is supportive of benefits from consuming activated water by individuals with various neurological and psychiatric illnesses. The major illnesses with anecdotal supportive data include Parkinson's disease, Alzheimer's disease, amyotrophic lateral sclerosis (ALS) [33], chronic fatigue syndrome (CFS), autism and bipolar psychoses. This is an enormous social toll of neuropsychiatric illnesses, including the tragedy of suicides, the indifference of criminal behaviors and the devastation of terrorism. These issues are not being effectively addressed with existing pharmaceuticals.

Mounting evidence also indicates that the ACE pathway is likely involved in higher levels of brain function, including mental clarity, attention span, cognition, self-awareness, mood and sleep. These functions can be diminished for various reasons, including infections with stealth adapted viruses, trauma, toxins, genetic anomalies and aging. Whatever the cause, they are likely to be noticeably improved by enhancing the ACE pathway. An aspect of effective brain function is the requirement for preventing non-selective over-firing from inappropriate regions of the brain. In the same way that the ACE pathway can function in the prevention of epileptic seizures [17], it appears to provide better coordination of higher brain functions. It could possibly do so by maintaining higher resting membrane potentials through diminishing the attraction of electrical charges spanning cell membranes.

Diseases of the brain may impair its proposed KELEA attracting capacity [16]. The consumption of KELEA activated water may possibly assist the brain in reaching a threshold required for a self-sustaining level of ACE pathway activation. At the other end of the spectrum of human behavior, it is reasonable to anticipate improvements in mental and physical performance of those seeking excellence in these endeavors. A swimmer once reported that upon using a device now known to activate water, that he was clearly able to swim farther underwater. This led to a provisional measure of improvements in the ACE pathway based on longer duration of breadth holding.

Individuals consuming activated water, while reporting improved restorative sleep, may also experience vivid, pleasant dreaming as if they have awakened a new resource for coping with the uncertainties of daily living. Sleep appears to allow a cleansing process leading to the removal of toxic metabolic byproducts released from neural cells [34]. The process is mediated by the intercellular passage of cerebrospinal fluid (CSF). It follows that this would be more efficient if the CSF is more permeable, allowing it to better penetrate between cells. A similar process may extend to an improved ability of activated body fluids to pass through tissues in the formation of lymph and the removal of metabolic waste products.

Cancer can be viewed as cells being deficient in one or other component and striving through replication and migration to obtain the missing component, at least for their progeny. Enhancing the cells' ACE pathway may help overcome the deficiency and relieve the stimulus for excessive growth and metastases. Apoptosis refers to a suicidal response that abnormal cells can undergo as a natural corrective mechanism in multicellular organisms. If apoptosis requires more energy than cellular replication, then arguably tumor cells persistence could simply reflect limited availability of energy. Given additional energy, the tumor may well lose its impetus to replicate or if intrinsically damaged will self-destruct by apoptosis. Whichever of these two explanations hold true, there are many examples of tumor regressions coinciding with therapies that are able to enhance the body's ACE pathway. 
It is particularly notable that tumor regression, presumably occurring through enhancement of the ACE pathway is an essentially painless process. Conventional cancer therapy with radiation and chemotherapy typically evokes inflammation with accompanying pain. Similarly, advanced efforts at immunotherapy of cancer, while occasionally highly successful, can lead to serious symptoms from "cytokine storms" and painful inflammation.

Possibly of even greater relevance than the treatment of cancer is the potential role of the ACE pathway in longevity. Normal aging has been attributed by some to a gradual failure of mitochondria production of cellular energy, leading to senescence and a failure to repair age related damage. Interestingly, tissue culture cells have been maintained in activated tissue culture medium, without any added feeding for over a year. As discussed in the next section, certain plants grown with the aid of activated water appear to have delayed senescence. Human populations living in certain regions of the world have extended life spans that have been attributed to consuming regional water. These locations include Lourds (France), Hunza (Pakistan), Nadana (India), Tlacote (Mexico), Marcial (Russia) and Teteven (Bulgaria).

\section{Agriculture and Other Farming Benefits of KELEA Activated Water}

Agricultural crops can become energy deficient from either insufficient photosynthesis or inadequate nutrient metabolism. The energy deficiency is commonly but not necessarily reflected in lower yields. It may, for example, be expressed mainly in reduced crop quality. In particular, there can be lower levels of secondary metabolites, which ordinarily provide valuable trace nutrients to humans and animals consuming healthy crops. The times to harvest certain crops can be reduced by using activated water, while there can be longer and more productive life cycles (delayed senescence) for other crops. Essentially, both rice and sugarcane have shown longer productivity cycles; a finding possibly relevant to longevity in humans. Growing crops in activated water can lead to a longer post-harvest shelf life, as was demonstrated with tomatoes [35]. Indeed, storing in KELEA activated water can improve the taste and delay the spoiling of meats, fruits and vegetables.

KELEA activated water obtained using cartridges of heated, ground volcanic rock (Kiko Technology) significantly increased the productivity of rice and sugarcane crops [35], even when harvested prior to the test crops reaching full maturity. The benefits were seen throughout the growth cycle, with improved root, stem and leaf structures. They were also noted in comparison rice fields following a high wind storm. The sturdier plants from the fields irrigated with activated water sustained far less damage than the plants in the control fields. Real time comparisons of treated and control crops have also been documented using drones equipped for near-infrared photography to monitor chlorophyll content [36].

Tests fields of both rice and sugarcane crops showed greater resistance to infections and to rodent infestations. Specifically, with activated water there was far milder and shorter lasting turgor virus infection in a treated rice field compared to the control field and similarly an absence of Downy mildew in the treated, but not in the control sugarcane field [35]. In addition, regression of pink rot fungal infections in palm trees has been achieved using KELEA activated water. Hydrogen bonding plays a critical role in the toxicity of certain fungal toxins [37]. It is possible, therefore, that the reduced hydrogen bonding resulting from activated water is inhibitory to certain fungal toxins. This was suggested by the rapid rise in numbers of aerobic bacteria, shortly after the application of the activated water to fungal infected palm trees (unpublished). Treatment of the palm trees with the activated water also stimulated fresh growth of the palms, supporting a possible additional role of the water in rejuvenation.

The increased permeability of KELEA activated water into the roots of plants has led in other studies to overall reductions in the amounts of water required to sustain optimal growth. Beyond water, the requirements for fertilizers and $\mathrm{pH}$ correcting soil amendments can also be reduced. Marked improvements have occurred with hydroponic cultivation of crops and in the colors of marketed flowers.

A growing problem for farmers is the accumulation of waste water, considered too toxic for further use on the farms or disposal in public waterways. Preliminary data indicate that highly toxic water be rendered reusable over time by the addition of activated water.

Improvements can also be obtained in farm animal husbandry as well as with domestic pets. Specifically, unpublished data from several test sites indicate more rapid maturation and increased egg laying by chickens, increased milk production by cows and reduced malodor of pig feces. The studies were performed with Kiko Technology and/or HB-101. The cultivation of farmed fish has similarly been improved with Kiko Technology. Contributing to the reported improvement was more uniform fish size with less growth-stunted fish that tend to 
be eaten by the larger fish. Inquiries have been received regarding activated water possibly improving the performance of race horses.

A partial summary of the major agricultural and animal husbandry applications of KELEA activated water is provided in Table 4. The listing is sufficiently compelling that all farmers should become involved in the use and further evaluation of KELEA activated water.

\section{Industrial and Environmental Benefits of KELEA Activated Water}

There are numerous major and minor industrial, environmental and household applications of KELEA activated water. Topping the list in terms of potential economic savings is the inclusion of KELEA related technologies in the activation of water used in electricity generating power plants. Of the total 355 billion gallons of water consumed in the United States in 2010, it is estimated that 161 billion gallons were used in power plants

(http://dx.doi.org/10.3133/cir1405). Water is essentially heated to generate high pressure steam, which provides mechanical force acting to spin a turbine. The spinning turbine rotates coiled wire within a magnetic field. This creates an electric current in the wire for further transmission to consumers. After being used to rotate the turbine, the steam is converted back to water by cooling with large quantities of additional water, which in turn are allowed to slowly cool by partial evaporation. Only liquid ammonia requires more energy to heat than does water. As noted earlier, in a closed space, less energy is required to heat activated water compared to regular water. In addition, the boiling point of KELEA activated water is reduced below $100^{\circ} \mathrm{C}$, making it easier to generate steam. Water used in power plants slowly corrodes metal pipes and also forms scale within the pipes [2]. Corrosion can lead to holes developing within the pipes, while scale reduces flow rates by narrowing the internal diameter of the pipes. Scale formation and corrosion also affect the turbines. KELEA activated water carries less of the calcium phosphate and other contaminating salts that contribute to scale formation than does regular water. It can also reverse the hydrogen bonding that allows scale material to form and to adhere to the pipes. Activated water may also lead to reduced friction between the flowing water and water pipes, either metal or plastic.

Large quantities of hot water are also generated for the heating of buildings in colder climates. The efficiency of this process can be increased using water with lowered specific heat value [2]. Water is also heated for cooking, bathing and numerous cleaning procedures, all of which are potentially easier with KELEA activated water. The enhanced industrial and household cleaning power of activated water is especially useful since it can penetrate small cervices more effectively than can regular water. Swimming pools and spars should similarly become less expensive to heat.

Refrigeration is a process of extracting heat from confined areas using the vapor of liquids with low boiling points. Pressure is used to re-condense the vapor back into the liquid phase. This generates heat that is commonly dissipated by being transferred to water. This type of cooling process was also shown to be more effectively achieved using activated water [2]. The term HVAC encompasses heating, ventilation and air conditioning activities, all of which are likely to be rendered more efficient using KELEA activated water.

Another major use of activated water is the ability to create more evenly dispersed suspensions of insoluble

Table 4. Farming applications of KELEA activated water.

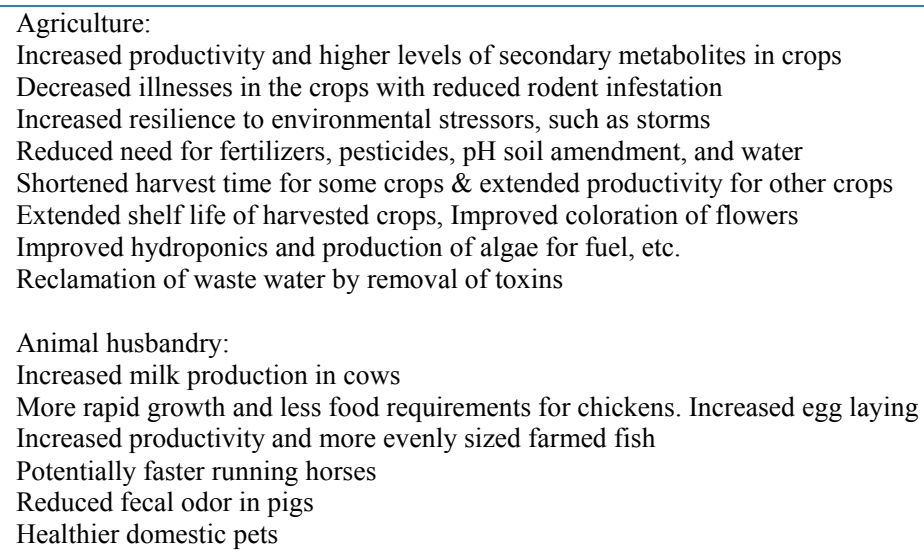


and colloidal materials. This is seen, for example, in the greater evenness of concrete and paints. With regard to concrete, the use of activated water can significantly increase its tensile strength and reduce its brittleness. Bakers report on making better bread and coffee made with activated water is said to be more flavorful. Certain enzymes and biochemical reactions can proceed more rapidly using activated water, while commensal microbes can seemingly gain competitive advantages over pathogenic microbes. Lawns are easier to water and pathway dirt easier to remove by washing with or in activated water. A particular use is the watering of golf course fairways with activated water to remove unsightly grassless areas of highly compact soil. Another potential use may be using more penetrating water in fracking for the production of oil.

As mentioned in the Agricultural section of this paper, water can become so contaminated with toxins that it is unsuitable for drinking or even for agriculture. Examples of contaminated water include abandoned water pools previously used to grow fish and shrimp. Other examples include pools of water collections adjacent to large ports and industrial facilities. Such water commonly emits offensive odors and may be carcinogenic. The addition of relatively small quantities of activated water can lead to the progressive activation of even large pools of water with precipitation of some of the contaminating toxic materials that can be removed by filtration or sedimentation. Activated water can similarly be used to help leach toxic materials out of contaminated soils. Indeed, this approach is underway to help remove Agent Orange from contaminated land sites in Vietnam.

As noted earlier, the upward flow of water in the foundations of buildings can be reversed using the Aquapol device [14]. The device comprises 3 outwardly projecting antennas, attached to a base plate. It does not require any external source of energy, but may acquire variable electrostatic charges from the Earth's electrical fields. Interestingly, there is local ionization of the air in the vicinity of the device (personal communication).

Vaporized water as a component of Brown's gas can be ignited and serve as a welding gas with some rather unique features [14]. For example, it will heat different materials to different temperatures. The welding can also be performed underwater. Brown's gas is also reported to be able to suppress the radioactive decay of ${ }^{60}$ Cobalt [14].

A summary of some of the major industrial and environmental applications of KELEA activated water are provided in Table 5. Again, the preliminary data are sufficiently compelling to encourage the testing of activated water in many industrial applications and to pursue the promise of more efficient KELEA delivering devices.

Table 5. Industrial applications related to KELEA activated fluids.

Power Plants: Less energy required to generate steam; more rapid cooling of steam; less scale and corrosion of pipes and of turbines

HVAC: Less energy required to heat water; more rapid cooling of condensed refrigerant; less scale and corrosion of pipes; less need for chemical additives

Drinking Water and Beverages: Healthier drinking water, other beverages and canned and bottled foods

Outdoor Watering: Watering of gardens, parks, golf courses, swimming pools, etc. Use of water for cleaning. Possibly improved fracking

Water Damage. Reversal of the upward seepage of water into building foundations

Miscellaneous: More efficient mixing of materials into water, including producing cement with higher tensile strength, more even paints. Efficient use of enzymes. Ignited Brown's gas used for welding, possible reduction of radioactivity decay

Activation of Other Fluids and Delivery to Consumers: Gasoline, diesel, liquid propane, ethanol

Activation of Gases and Delivery to Consumers: Propane, hydrogen, natural gas

Reduced resistance to electrical current. Yet to be proven

Manufacturing of water activating devices, as either containers for water activating compounds or as items to concentrate KELEA for its transfer to nearby fluids

Manufacturing of devices and/or other approaches to support the capacity of individuals to directly attract KELEA into their body 


\section{Matching Activation Method to Application}

The choice between different methods of activation is influenced, in part, by the desired application of the activated water. There can also be personal preferences, for example between consuming moringa oleifera [38] or cocoa activated water. An important area of research is to evaluate the suitability of various water activating methods when the water is intended for particular uses. A major distinction will be between adding enerceuticals $^{\mathrm{TM}}$ or locally concentrating KELEA using various devices. An advantage of the latter is that the devices can also be directly tested in the treatment of patients. They also will meet with easier regulatory approvals in activating water for human consumption.

Water activating compounds can either be soluble, with the possibility of their being removed by filtration from the activated water, or insoluble and placed in retrievable cartridges. Issues to be considered include cost, quantity of water needed, the intended use of the water, how quickly does it need to be activated and whether there will be delays in use. Although, some of the least expensive water activating methods are relatively slow acting, this can still be appropriate when long-term activation is envisioned. For example, agricultural applications and heating of recirculating water systems can proceed relatively slowly and yet still be effective. Undoubtedly, major improvements will occur in adopting and comparing different methods of water activation for use in the various applications discussed in the paper.

\section{Activation of Other Fluids}

KELEA can also be applied to the activation of other fluids, again with potentially enormous beneficial consequences. For example, ethanol [1] and alcoholic beverages are easily activated using any of the many methods applicable to water. Small quantities of activated ethanol can be useful as an activating additive to regular water. Gasoline can be activated [1] and this can be done either before fueling or within the gas tank. Several years ago, I was informed that small quantities of HB-101 added to the fuel tank would significantly improve fuel efficiency. This approach has the disadvantage of using a water based product, as does the use of Brown's gas obtained by electrolysis. This concern does not apply to the addition of soluble granules, especially when contained within removable cartridges. Magnets that spin with the flow of gasoline have been placed in fuel lines with reported benefits, which can now be attributed to KELEA activation of the gasoline. Another reported method of improving fuel economy, is the strapping of a container of activated water to the outside of the fuel line. In addition to improved mileage, it is commonly stated that the car engines, as well as lawn mower engines run cooler. It seems that with activated gasoline, more of the chemical energy of combustion is transferred to mechanical, rather than to heat energy. Marked reductions have also been noted in carbon monoxide emissions. It is also likely there will be less scale formation and/or corrosion in the metallic fuel lines. Activation of diesel fuel should also improve combustion and provide major benefits in transportation and heating industries.

While fluids are commonly considered as comprising liquids, the term also applies to gases. Increased vapor pressure of KELEA activated ethanol has been shown [1] and is consistent with KELEA acting on gas molecules. It will not be surprising, therefore, that the various activation techniques developed for water and more recently for gasoline, will find application in increasing heat generation (expressed as British Thermal Units) in the combustion of natural gas and propane. Even air is a fluid and presumably, therefore, subjected to KELEA mediated increases in its kinetic activity and possibly its degree of ionization. Finally, it is not unreasonable to consider that the movement of electrons in electrical circuits is comparable to fluid moving against electrostatic resistance. Applying KELEA to electrical circuitry may, therefore, possibly lead to reduced resistance and greater efficiency of electricity usage. These and other far reaching concepts should not go unexplored, but are clearly beyond the capabilities of a single institution.

\section{The Value of Coordinating Studies}

Multiple methods for activating water and other fluids with KELEA are available. There are also numerous existing and potentially new health, agricultural and industrial applications for using the KELEA activated fluids. Clearly different expertise is needed for further developing the activation methods and particularly for quantitatively assessing the various proposed uses of the activated fluids. Central coordination of the studies, along with pursuing a better scientific this coordination will be useful in providing constructive feedback among participating organizations. This coordination will also help ensure that advances are quickly conveyed to various parts of the world for rapid implementation. 
It is, therefore, proposed that with centralized support, individual philanthropic organizations could choose a project appropriate to their size, technical expertise and prior experience. Essentially, the philanthropic organization would choose from among the various projects included in Tables 3-5 or pursue a related project. The centralized technical hub would function in an advisory manner and also assist in the sharing of new insights and other developments. It would also work to improve upon the scientific understanding of KELEA.

Within the sphere of human illnesses, there already exist many organizations devoted to the study of particular diseases. With large patient followings, these specific disease oriented organizations could quickly compare the benefits of KELEA activated water with those of current pharmaceuticals [39] [40]. It may even be shown that certain commonly used pharmaceuticals actually achieve their main benefit through water activating activity, rather than through their biochemical effects. There should be no delays in initiating clinical studies using KELEA activated water in humans and animals to help control the increasing costs of pharmaceuticals. Far more importantly, the studies will likely achieve markedly improved health throughout the world.

Extending KELEA activation to industrial and agricultural applications and including additional fluids in industrial studies will similarly provide a boost to the world's economy. Attempting to pursue restrictive, competitive business models with minimal disclosure of technical information would be a disservice to humanity. Again, there could be a cooperative endeavor involving philanthropic and other organizations collectively geared towards the optimization of applications beyond human and animal health. This type of collective undertaking could provide a powerful alternative to current industrial, economic and military based competitions.

\section{Summary}

Enormous value can potentially be rapidly realized by the widespread utilization of KELEA activated water in health, agriculture, and industry. Philanthropic organizations could play an important role as team leaders in their areas of experience and expertise. A centralized, coordinating program is envisioned to assist with the ready exchange of technical information and the dissemination of proven advances. Funding beyond what the philanthropic organizations may be able to provide should come from governments. Some economists suggest that for really important purposes, governments should simply print new money, essentially as a tax on capital. Hopefully, this article will help lay the groundwork for a renewed sense of political and humanitarian purpose.

\section{Acknowledgements}

The Institute of Progressive Medicine is a component of MI Hope Inc., a non-profit public charity. The author is particularly thankful to Mr. Nobo Azuma, who marketed HB-101; James Osugi, Founder and President of Kiko Technology; David Christner, manufacturer of Enercel, Geoff McMahon, supplier of humic/fulvic acids and many others for kindly sharing data and providing reagents.

Conflict of Interest: None.

\section{References}

[1] Martin, W.J. (2015) KELEA: A Natural Energy That Seemingly Reduces Intermolecular Hydrogen Bonding in Water and Other Liquids. Open Journal of Biophysics, 5, 69-79. http://dx.doi.org/10.4236/ojbiphy.2015.53006

[2] Martin, W.J. (2015) Improved Efficiency of Heat Exchange Using KELEA Activated Water. Open Journal of Energy Efficiency, 4, 36-43.

[3] Martin, W.J. (2015) Therapeutic Potential of KELEA Activated Water. International Journal of Complementary \& Alternative Medicine, 1, Article ID: 00001.

[4] Fischer, P. (2012) What Is Homeopathy? An Introduction. Frontiers in Bioscience (Elite Edition), 4, 1669-1682. http://dx.doi.org/10.2741/E489

[5] Martin, W.J. (2014) Stealth Adapted Viruses; Alternative Cellular Energy (ACE) \& KELEA Activated Water. AuthorHouse In., Bloomington, 321.

[6] Brown, Y. http://www.eagle-research.com/cms/store/browns-gas

[7] Cayce, E. http://www.cayce.com/violet_ray_true_health.html

[8] Silver, N. (2011) The Rife Handbook of Frequency Therapy and Holistic Health. Desert Gate Productions Az., Phoenix, 742.

[9] Lakhovsky, G. (1996) The Secret of Life. Cosmic Rays and Radiations of Living Beings. Translated by Clement, M., 
Health Research, Pomeroy, 213.

[10] Martin, W.J. (2015) Interacting Light Paths Attract KELEA (Kinetic Energy Limiting Electrostatic Attraction) and Can Lead to the Activation of Water. Open Journal of Biophysics, 5, 115-121. http://dx.doi.org/10.4236/ojbiphy.2015.54010

[11] Martin, W.J. (2015) Interactive Electric Fields Attract KELEA (Kinetic Energy Limiting Electrostatic Attraction) and Can Lead to the Activation of Water. International Journal of Complementary \& Alternative Medicine, 1, Article ID: 00034.

[12] Reich, W. (1948) The Cancer Biopathy. The Discovery of the Orgone. Vol. 2, Translated by White, A., Higgins, M. and Raphael, C.M., Farrar, Straus and Giroux, New York, 433.

[13] Roerich, V. http://www.angelfire.com/tn/moonlodge/aboutintra.html

[14] Mohorn, W. http://www.aquapol.co.uk/index.php?go=science/energy

[15] Pleasanton, A. (1877) The Influence of the Blue Ray of the Sunlight and of the Blue Color of the Sky. Remsen \& Haffelfinger, Claxton, 185.

[16] Martin, W.J. (2015) Is the Brain an Activator of the Alternative Cellular Energy (ACE) Pathway? International Journal of Complementary \& Alternative Medicine, 1, Article ID: 00002.

[17] Martin, W.J. (2014) Alternative Cellular Energy (ACE) Activation as Natural Therapy for Autism. In: Stealth Adapted Viruses; Alternative Cellular Energy (ACE) \& KELEA Activated Water. AuthorHouse In., Bloomington, 87-102.

[18] Polack, G.H. (2013) The Fourth Phase of Water: Beyond Solid Liquid and Vapor. Ebner and Sons, Seattle, 357.

[19] DeMeo, J. (2014) Spectrographic Signatures in Water Induced by Radiant Fields from Enclosures of Various Materials. Presented at Conference on the Physics, Chemistry and Biology of Water, Bulgaria. www.orgonelab.org

[20] Martin, W.J. (2014) KELEA Activated Water-Enhancing the Alternative Cellular Energy (ACE) Pathway. In: Stealth Adapted Viruses; Alternative Cellular Energy (ACE) \& KELEA Activated Water, AuthorHouse In., Bloomington, 115144.

[21] Emoto, M. (2011) The Hidden Messages in Water. Atria books, New York, 200.

[22] Wells, J.C. (2013) Obesity as Malnutrition: The Dimensions beyond Energy Balance. European Journal of Clinical Nutrition, 67, 507-512. http://dx.doi.org/10.1038/ejen.2013.31

[23] Batmanghelidj, F. (2008) Your Body’s Many Cries for Water. Global Health Solution, Falls Church, 196.

[24] Martin, W.J. (2014) Stealth Adaptation of Viruses: Review and Updated Molecular Analysis on a Stealth Adapted African Green Monkey Simian Cytomegalovirus (SCMV). Journal of Human Virology \& Retrovirology, 1, Article ID: 00020. http://dx.doi.org/10.15406/jhvrv.2014.01.00020

[25] Martin, W.J. (2015) Stealth Adaptation of Viruses: Implications for Therapy and for Potential Toxicity of Vaccines. Journal of Virology and Current Research, 1, Article ID: 555551.

[26] Martin, W.J. (2015) Stealth Adapted Viruses. A Bridge between Molecular Virology and Clinical Psychiatry. Open Journal of Psychiatry, 5, 311-319.

[27] Martin, W.J. (2015) Stealth Adapted Viruses-Possible Drivers of Major Neuropsychiatric Illnesses Including Alzheimer's Disease. Journal of Neurology \& Stroke, 2, Article ID: 00057.

[28] Dubrov, V., Dubrova, T., Christner, D., Laurent, D. and Martin, W.J. (2015) Alternative Cellular Energy Based Therapy Using Enercel ${ }^{\mathrm{TM}}$ in Advanced AIDS Patients Co-infected with Tuberculosis and Treated in Chernigov, Ukraine. Journal of Human Virology \& Retrovirology, 2, Article ID: 00061.

[29] Martin, W.J. and Stoneburner, J. (2014) Alternative Cellular Energy (ACE) Pathway Activation as the Mode of Action of Neutral Red Dye Phototherapy of Human Viruses. Journal of Human Virology \& Retrovirology, 1, Article ID: 00019. http://dx.doi.org/10.15406/jhvrv.2014.01.00019

[30] Izaguirre, R.R., Guzman, M.R., Fuentes, R.C., Mena, C.E., Penate, E. and Martin, W.J. (2014) Alternative Cellular Energy Based Therapy of Childhood Diarrhea. In: Stealth Adapted Viruses; Alternative Cellular Energy (ACE) \& KELEA Activated Water, AuthorHouse In., Bloomington, 103-112.

[31] Dubrov, V., Dubrova, T., Suhareva, V., Christner, D., Baiamonte, J. and Laurent, D. (2012) Efficacy of Treatment with Enercel for New-Onset, Presumed Drug-Sensitive and Confirmed Multidrug Resistant Pulmonary Tuberculosis. USAID/ Ukraine, Tuberculosis, Lung Infections and HIV, 1, 85-91.

[32] Martin, W.J. and Palmer, S.B. (2010) Regenerative Wound Healing Using Copper-Silver Citrate Composition. US Patent Application No. 20100099758.

[33] Liang, S., Christner, D., Du Laux, S. and Laurent, D. (2011) Significant Neurological Improvement in Two Patients with Amyotrophic Lateral Sclerosis after 4 Weeks of Treatment with Acupuncture Injection Point Therapy Using Enercel. Journal of Acupuncture and Meridian Studies, 4, 257-261. http://dx.doi.org/10.1016/j.jams.2011.09.017 
[34] Mendelsohn, A.R. and Larrick, J.W. (2013) Sleep Facilitates Clearance of Metabolites from the Brain: Glymphatic Function in Aging and Neurodegenerative Diseases. Rejuvenation Research, 16, 518-523. http://dx.doi.org/10.1089/rej.2013.1530

[35] Martin, W.J. (2014) KELEA Activated Water Leading to Improved Quantity \& Quality of Agricultural Crops. Advances in Plants \& Agriculture Research, 2, Article ID: 00033.

[36] Martin, W.J. (2015) Use of Drone to Monitor Enhanced Production of Chlorophyll in Agricultural Crops Resulting From KELEA Activation of Water.

https://www.researchgate.net/publication/274193905_Use_of_Drone_to_Monitor_Enhanced_Production_of_Chloroph yll in Agricultural_Crops Resulting_From_KELEA_Activation of Water\#full-text

[37] Chaudhary, P., Shank, R.A., Montina, T., Goettel, J.T., Foroud, N.A., Hazendonk, P. and Eudes, F. (2011) Hydrogen-Bonding Interactions in T-2 Toxin Studied Using Solution and Solid-State NMR. Toxins, 3, 1310-1331. http://dx.doi.org/10.3390/toxins3101310

[38] Martin, W.J. (2015) Do the Benefits of Moringa oleifera Trees Extend to KELEA Activation of Water? Advances in Plants \& Agriculture Research, 2, Article ID: 00036. http://dx.doi.org/10.15406/apar.2015.02.00036

[39] Martin, W.J. (2015) Alternative Cellular Energy. A Unifying Concept in Complementary Alternative Medicine. International Journal Complementary \& Alternative Medicine, 1, Article ID: 00022.

[40] Martin, W.J. (2016) Deconstructing Medicine. The Alternative Cellular Energy Pathway. British Journal of Medicine \&Medical Research, 11, 1-6. http://dx.doi.org/10.9734/BJMMR/2016/21484

\section{Abbreviations}

ACE—alternative cellular energy;

$\mathrm{CSF}$ - cerebrospinal fluid;

ICE - insufficiency of cellular energy;

KELEA - kinetic energy limiting electrostatic attraction;

HIV - human immunodeficiency virus. 